Cad.Est.Ling., Campinas, (40):53-60, Jan./Jun. 2001

\title{
PADRÕES INICIAIS NA AQUISIÇÃO DO SISTEMA DE SONS DO PORTUGUÊS: CARACTERÍSTICAS UNIVERSAIS E ESPECÍFICAS ${ }^{1}$
}

\author{
ELIZABETH REIS TEIXEIRA \\ (Universidade Federal da Bahia)
}

\begin{abstract}
Adopting a strictly motor approach to linguistic organization, the theoretical proposal called Molde $e$ Conteúdo (Davies \& Macneilage 1995; Macneilage \& Davies 1966) argues that the first child production akin to adult speech - canonical babbling - can be seen as a motor correlate of the molde silábico. Moldes constitute a rhythmic basis catalyzing specific syllabic or segmental sequences. The independent motor control of such elements emerges gradually from jaw/tongue synergies with which the child initiates a type of vocal production similar to the adult's. In the acquisition of speech production skills, then, changes in the quality of sounds in an utterance are initially based in changes in the amplitude of the jaw opening/closing cycle, i.e., they determine intra-syllabic organization. Syllables sequences, in turn, comprise vocalic and consonantal qualities produced in homorganic articulation places (i.e. front consonants with front vowels; back consonants with back vowels), as demonstrated child data derived from six different linguistic communities - English, Swedish, French, Japanese (in the Stanford database), Brazilian Portuguese (Teixeira \& Davies 1999) e Equatorial Quichua (Gildersleeve-Neumann, doctoral dissertation in preparation, U. Texas, Austin). In general, the data on Brazilian Portuguese acquisition are comparable to production universal patterns reported for children in different languages, but also show specific characteristics due to the ambient language pressure.
\end{abstract}

\section{INTRODUÇÃO}

A unidade mais básica da linguagem é a palavra - o mínimo acoplamento independente de significado e de estrutura sonora. Mas, qual é a origem deste acoplamento? Além daquelas poucas palavras que são indubitavelmente onomatopaicas, os linguistas consideram este acoplamento como basicamente arbitrário - isto é, eles acreditam que a estrutura conceitual de uma palavra não impõe uma estrutura sonora determinada a sua forma falada, independentemente da língua. Contudo, se a estrutura conceitual, ou o significado, de uma palavra não determina seu padrão sonoro, então, o quê determina? Estranhamente, pouca atenção tem sido dada à origem da estrutura interna das formas das palavras. Supõe-se que existam fatores determinantes inerentes à própria produção de estruturas sonoras nas palavras, além de sua notória tendência de alternar entre consoantes e vogais para formar sílabas (ex.TOMA-TE).

1 Trabalho apresentado na Mesa Redonda - Processamento Fonético-Fonológico e Lexical, Encontro da ANPOLL- Junho/ 2000, Universidade Federal Fluminense, Niterói. 


\section{OS PADRÕES INTRA-SILÁBICOS}

Estudos estatísticos foram conduzidos sobre o balbucio de 6 bebês e sobre as primeiras palavras de 10 crianças em um meio-ambiente falante do inglês. Quatro padrões organizacionais potencialmente universais emergiram. Três deles eram padrões de co-ocorrência intra-silábica $(\mathrm{CV})$ :

- consoantes labiais com vogais centrais,

- consoantes coronais (antero-linguais) com vogais anteriores,

- e consoantes dorsais (postero-linguais) com vogais posteriores.

A Figura 1, abaixo, mostra uma visão esquemática do componente articulatório do aparato da fala, no qual as três setas simbolizam os 3 padrões intra-silábicos de coocorrência da CV.

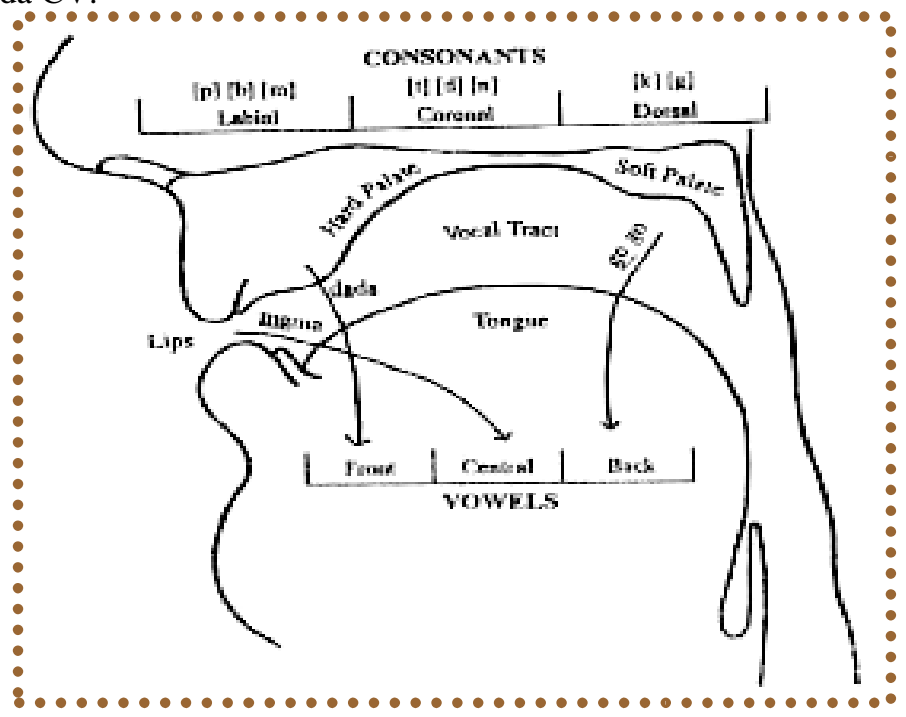

Figura 1: VISÃO ESQUEMÁTICA DO COMPONENTE ARTICULATÓRIO DO APARATO DA FALA

(Fonte: MacNeilage \& Davis 2000) As consoantes labiais envolvem oclusão labial; as consoantes coronais envolvem oclusão entre a lâmina da língua e a parte anterior do trato oral (região da arcada dental superior e regiões alveolar e pós-alveolar); as consoantes dorsais envolvem oclusão entre o dorso da língua e a região velar (do palato mole ou véu palatino).

Três estudos adicionais usando a mesma metodologia específica revelaram os mesmos efeitos em grupos de 5 bebês franceses, suecos e japoneses (Boysson-Bardies and Vihman 1991), 7 bebês de um ambiente Quichua Equatoriano (GildersleeveNeumann, Tese de doutorado em andamento) e uma de duas crianças de um ambiente de Português Brasileiro (Teixeira, 1997; Teixeira e Davis 1999, 2001). Se existe, de fato, uma tendência para o Balbucio e, em menor escala, as Primeiras Palavras serem semelhantes em culturas distintas, esses padrões parecem ser realmente universais nas crianças. 


\begin{tabular}{|c|c|c|c|c|c|c|c|c|c|c|c|c|c|c|c|c|c|c|}
\hline \multicolumn{2}{|c|}{ FRANCÊS } & \multicolumn{3}{|c|}{ SUECO } & \multicolumn{3}{|c|}{ JAPONÊS } & \multicolumn{3}{c|}{ INGLÊS } & \multicolumn{3}{c|}{ QUICHUA } & \multicolumn{3}{c}{$\begin{array}{c}\text { PORTUGUÊS } \\
\text { BRASILEIRO }\end{array}$} \\
\hline C & L & D & C & L & D & C & L & D & C & L & D & C & L & D & C & L & D \\
\hline
\end{tabular}

\begin{tabular}{l|l|l|l|l|l|l|l|l|l|l|l|l|l|l|l|l|l|l}
\hline $\mathrm{A}$ & 1.18 & & & 1.17 & & & 1.21 & & & 1.27 & & & 2.61 & & & 1.01 & & \\
\hline $\mathrm{C}$ & & 1.16 & & & 1.24 & & & 1.22 & & & 1.17 & & & 1.62 & & & 1.05 & \\
\hline $\mathrm{P}$ & & & 2.43 & & & 1.38 & & & 1.11 & & & 1.23 & & & 2.13 & & & 1.47 \\
\hline
\end{tabular}

\begin{tabular}{|c|c|c|c|c|c|c|c|c|c|c|c|c|c|c|c|c|}
\hline$\overline{\mathbf{A}}$ & 1.61 & & & \multirow{3}{*}{ Em andamento } & 1.00 & & & 1.16 & & & 1.26 & & & .91 & & \\
\hline $\mathbf{C}$ & & 1.19 & & & & .73 & & & 1.02 & & & 1.14 & & & 1.04 & \\
\hline $\mathbf{P}$ & & & \begin{tabular}{|l|l}
1.62 \\
\end{tabular} & & & & \begin{tabular}{|l|l}
1.17 \\
\end{tabular} & & & 1.50 & & & $\begin{array}{l}1.54 \\
\end{array}$ & & & 1.22 \\
\hline
\end{tabular}

(Fonte: Davis, MacNeilage, Gildersleeve-Newmann \& Teixeira (1999) ${ }^{2}$

${ }^{2}$ Os resultados estão expressos em forma da taxa entre freqüências observadas e esperadas (i.e acima do acaso, above chance) em cada célula (o valor mínimo esperado é de 1.0). As frequiências esperadas para cada uma das três categorias vocálicas foram calculadas a partir da frequiência total da vogal no corpus. Isto é, se $40 \%$ das vogais no corpus são vogais centrais, então se esperaria que $40 \%$ de todas as vogais em cada ambiente consonantal fossem centrais. As associações esperadas aparecem destacadas na diagonal. 
De acordo com a teoria Moldes/Conteúdo (Davies \& Macneilage 1995; Macneillage \& Davis 1996, 2000), em todos os três padrões de co-ocorrência de consoante-vogal, a seqüência CV manifesta-se através da alternância boca fechada/boca aberta, i.e., através de um movimento básico, ou molde bifásico, da oscilação mandibular: em que existe elevação para as consoantes e depressão para as vogais. Na co-ocorrência de CV labial-central realiza-se o "molde puro" (o molde parece ser a única causa da combinação, vez que não existem, aí, movimentos linguais). Nestes casos, uma fase de fechamento da oscilação mandibular (agindo independentemente) estaria produzindo o fechamento labial, enquanto uma fase aberta da oscilação (também de forma independente) estaria produzindo vogais centrais, partindo a língua de uma posição mais próxima a sua posição de descanso no centro da boca. Esta forma simples pode ter sido o tipo proto-silábico mais básico. O uso destes moldes deve ter sido o primeiro estágio na evolução da fala. Em um momento subseqüente da evolução do "conteúdo", deve ter se desenvolvido a capacidade mais avançada para programar moldes sucessivos com diferente consoantes e vogais, freqüentemente envolvendo movimentos linguais predominantemente na direção da consoante para a vogal.

\section{O PADRÃO INTER-SILÁBICO}

O quarto padrão, inter-silábico, não está presente no balbucio, mas emerge durante o período das primeiras palavras. Sete relatos de 5 comunidades linguiísticas revelam a tendência para se começar uma palavra com consoante oclusiva labial, seguida de vogal e, depois, de uma consoante oclusiva coronal: uma seqüência LVC, (ou LVCV, como ocorre em Português) (Macneillage \& Davis 2000).

O padrão LC é o primeiro passo sistemático na direção da diferenciação dos moldes inter-silábicos na fala das crianças. No balbucio, os bebês tendem a simplesmente repetir a mesma sílaba ([bababa]) - um caso de reiteração do molde. Mas, de acordo com o princípio bastante aceito na teoria fonológica do contorno obrigatório, as línguas tendem a favorecer um padrão inter-silábico discontínuo - que requer que o falante produza uma consoante e/ou uma vogal diferente em sílabas sucessivas. A produção da sequiência LC pelas crianças é um evento marcante porque é o primeiro passo sistemático, depois de uma repetição relativamente obrigatória do ciclo CV para uma não-repetição compulsória, ou variegação. 


\begin{tabular}{|c|c|c|c|c|c|c|c|c|}
\hline & & \multicolumn{3}{|c|}{$0 ; 12-0 ; 23$} & \multicolumn{3}{|c|}{$0 ; 24-0 ; 36$} & \\
\hline PADRÃO & S1 & S2 & $\begin{array}{c}\text { SUB } \\
\text { TOTAL } \\
(\%)\end{array}$ & S1 & S2 & $\begin{array}{c}\text { SUB } \\
\text { TOTAL } \\
(\%)\end{array}$ & $\begin{array}{c}\text { MÉDIA } \\
(\%)\end{array}$ & $\begin{array}{l}\text { CDI }^{3} \\
\text { BP }\end{array}$ \\
\hline
\end{tabular}

\begin{tabular}{c|c|c|c|c|c|c|c|c}
\hline $\mathbf{L}+\mathbf{C}$ & 70 & 31 & $\mathbf{1 9}$ & 59 & 60 & $\mathbf{2 3}$ & $\mathbf{2 1}$ & $\mathbf{2 0 1}(\mathbf{2 5 \%})$ \\
\hline $\mathbf{L}+\mathbf{D}$ & 14 & 5 & $\mathbf{3}$ & 26 & 18 & $\mathbf{8}$ & $\mathbf{6}$ & $\mathbf{5 5}(\mathbf{7 \%})$ \\
\hline $\mathbf{C}+\mathbf{D}$ & 15 & 4 & $\mathbf{3}$ & 15 & 11 & $\mathbf{5}$ & $\mathbf{4}$ & $\mathbf{4 2}(5 \%)$ \\
\hline $\mathbf{C}+\mathbf{L}$ & 13 & 15 & $\mathbf{5}$ & 20 & 21 & $\mathbf{8}$ & $\mathbf{7}$ & $\mathbf{7 0}(\mathbf{9 \%})$ \\
\hline $\mathbf{D}+\mathbf{L}$ & 19 & 0 & $\mathbf{3}$ & 14 & 8 & $\mathbf{4}$ & $\mathbf{4}$ & $\mathbf{5 0}(6 \%)$ \\
\hline $\mathbf{D}+\mathbf{C}$ & 63 & 3 & $\mathbf{1 2}$ & 46 & 29 & $\mathbf{1 4}$ & $\mathbf{1 3}$ & $\mathbf{1 1 1}(\mathbf{1 4 \%})$ \\
\hline TOTAL & $\mathbf{4 0 6}$ & $\mathbf{1 3 7}$ & $\mathbf{5 4 3}$ & $\mathbf{2 7 1}$ & $\mathbf{2 5 0}$ & $\mathbf{5 2 1}$ & $\mathbf{1 0 6 4}$ & $\mathbf{7 9 9}$
\end{tabular}

TABELA 2: PADRÕES DE VARIEGAÇÃO NAS DUAS CRIANÇAS: a) DE 0;12 A 0;23 - b) DE 0;24 A 0;36 E c) EM TODO O CORPUS; E NO CORPUS DO CDI EM PB

(Fonte: Teixeira \& Davis 1999, 2001)

Esta tendência, chamada de "Anteriorização" é tão forte em algumas crianças que elas até a produzem mesmo quando a palavra-alvo que querem enunciar tem a seqüência oposta (CL), como, por exemplo, "POT" em vez de "TOP" No estudo de Macneillage \& Davis (1996), 9 de 10 crianças de ambiente de língua inglesa exibiram este padrão, sendo que o décimo não demostrou nenhuma preferência. A média da razão entre o número de seqüências de LC e o número de seqüências total foi 2,55.

A Tabela 2, acima, mostra os dados sobre os padrões inter-silábicos de reduplicação e variegação para duas crianças falantes do Português Brasileiro investigadas longitudinalmente. (Teixeira 1997; Teixeira e Davis 1999, 2001) Tanto os padrões reduplicados como os variegados coexistiram durante todo o período analisado. No primeiro período, os padrões reduplicados (55\%) foram mais numerosos que os variegados $(45 \%)$. No segundo período etário, ocorreram $38 \%$ de padrões reduplicados e $62 \%$ de sequiências variegadas. No total, a freqüência de padrões variegados $(55 \%)$ foi ligeiramente mais elevada do que a dos reduplicados (45\%). A reduplicação do traço coronal $(\mathbf{C}+\mathbf{C}, 30 \%)$ foi o padrão mais freqüente de associação entre consoantes na cabeça de duas sílabas consecutivas tanto em cada período separadamente, como na soma dos dois períodos. Em seguida, os padrões variegados $\mathbf{L}+\mathbf{C}(21 \%), \mathbf{D}+\mathbf{C}(13 \%)$ e o padrão reduplicado $\mathbf{L}+\mathbf{L}(11 \%)$ tiveram as taxas de ocorrência mais elevadas. S1 usou o padrão variegado $\mathbf{D + C}$ e o padrão reduplicado D+D com muito mais freqüência do que S2 no primeiro período. No segundo estágio, a diferença entre as duas crianças decresce.

O padrão variegado mais comum foi o $\mathbf{L}+\mathbf{C}(21 \%)$. É, sem dúvida o padrão mais freqüente dos dados destas crianças. Este padrão também já foi notado em 9 crianças

3 Adaptação de um dos protocolos dos Inventários de Desenvolvimento Comunicativo MacArthur (CDI) (Fenson, Dale, Reznick, Bates, Hartung, Pethicks \& Reilley 1992), para o Português, a fim de verificar a influência da língua ambiente. 
aprendendo inglês e em dissílabos de 9 entre 10 línguas pesquisadas (MacNeilage, Davis, Kinney \& Matyear, 1999). A Tabela 11 também mostra a análise da adaptação do $C D I$ ao PB. C+C (26\%) é a forma mais reduplicada e $\mathbf{L}+\mathbf{C}(25 \%)$ é a forma variegada mais freqüente, o que é bastante compatível com os resultados dos dois sujeitos. Em ambas as crianças e na adaptação do $C D I$ ao $\mathrm{PB}$, a segunda sequiência de variegação mais recorrente é $\mathbf{D + C}$.

No primeiro período etário, portanto, $55 \%$ de formas reduplicadas foram encontradas. Estas formas reduplicadas decresceram para $38 \%$ no segundo período, demonstrando um grau de variegação mais aproximado ao encontrado no uso da língua. Em geral, a adaptação do $C D I$ ao PB apresenta $34 \%$ de reduplicação, se aproximando das frequiências encontradas no segundo período dos dois sujeitos. A harmonia consonantal, freqüentemente descrita como um efeito da aquisição mais inicial, parece coincidir com o que ocorre nos dados destas crianças no primeiro período.

Embora o padrão LC seja a primeira estrutura enunciativa variegada nas Primeiras Palavras de crianças falantes do Português, o que confirma a tendência já relatada na literatura, a recorrência do padrão $\mathrm{D}+\mathrm{C}$ nos dados das crianças (13\%) parece refletir sua frequiência relativa na língua ambiente, a julgar-se por sua ocorrência na adaptação do $C D I$ ao PB (14\%). Neste caso, os efeitos de produção parecem ser dominantes para explicar a frequiência relativa de $\mathrm{L}+\mathrm{C}$, também encontrada no inglês e em outras línguas. Já o efeito secundário do aparecimento de D+C tão cedo parece ser explicável a partir da forte presença da língua ambiente na diversificação inicial da ordem serial dos elementos consonantais.

Os padrões dominantes no estudo de duas crianças aprendizes de PB de 12 a 36 meses (Teixeira 1997; Teixeira e Davis 1999, 2001) afirmam a primazia dos efeitos do sistema de produção já observados em outros estudos sobre este período. Labiais e coronais são tão proeminentes como a vogal central baixa. A reduplicação ocorre com maior freqüência no período mais inicial, corroborando os achados de outros estudos sobre o período. A indução de complexidade em sequiências variegadas pelo uso de $\mathrm{L}+\mathrm{C}$ foi confirmada tanto nos dados das crianças, como nos alvos freqüentemente usados por crianças aprendizes de PB demonstrados através da adaptação do CDI ao PB. Contudo, a evidência a respeito de influências perceptuais relacionadas à língua ambiente foi notada através da frequiência relativa das dorsais e do padrão variegado $\mathrm{D}+\mathrm{L}$, aspectos estes coincidentes com as freqüências observadas na língua ambiente. A presença destes tipos de efeitos sugere que os efeitos de produção não conseguem dar conta de explicar, com exclusividade, o que ocorre na aquisição mais inicial. Até mesmo nas formas lexicais mais iniciais, as evidências sobre os efeitos perceptuais do sistema de sons circundante fazem-se presentes no desenvolvimento e devem ser levadas em conta para o entendimento global da natureza da aquisição inicial do sistema de sons pela crianças. 


\section{CONCLUSÃO}

A explicação para a ocorrência destes padrões segundo Macneillage \& Davis (2000), no que diz respeito aos três padrões de co-ocorrência de CV, relaciona-se à alternância entre os estágios aberto e fechado do ciclo mandibular durante a produção da fala. A emergência, contudo, do padrão LC na fala infantil representa um marco em relação à mudança sistemática do uso obrigatório da repetição do mesmo ciclo CV para uma utilização quase obrigatória da não-repetição. A principal diferença entre o efeito da seqüência LC e os efeitos das co-ocorrências de Consoante + Vogal é que os padrões CV são contínuos, i.e. envolvem relações entre sons adjacentes. Estes padrões representam um único efeito biomecânico que opera em sons que se avizinham, como no caso dos dois padrões linguais de CV (o coronal-anterior e o dorsal-posterior). $\mathrm{O}$ padrão LC, contudo, é discontínuo: seus dois componentes separam-se, no eixo temporal, pela interveniência da vogal. Uma possível explicação para a existência e recorrência desta discontinuidade é a maior facilidade articulatória exigida para a produção de uma consoante labial do que para uma coronal. Além disso, dois fatos aquisicionais sugerem que as consoantes labiais são mais fáceis para as crianças do que as coronais: aparecem primeiro tanto no Balbucio como nas Primeiras Palavras; aparecem em maior quantidade nas produções de crianças surdas (caso em que a a sensibilidade à força dos padrões da língua ambiente praticamente inexiste).

Apesar destas motivações produtivas, a recorrência do padrão DL na aquisição do Português, mesmo nos estágios aquisicionais iniciais, coincidente com a alta taxa de ocorrência destes aspectos na língua ambiente, evidencia a força das influências perceptuais. A presença destes efeitos sugere que as características de produção não conseguem dar conta de explicar, com exclusividade, o que ocorre na aquisição mais inicial. Até mesmo nas formas lexicais mais iniciais, as evidências sobre os efeitos perceptuais do sistema de sons circundante fazem-se presentes no desenvolvimento e devem ser levadas em conta para o entendimento global da natureza da aquisição inicial do sistema de sons pela crianças.

\section{REEFERÊNCIAS}

BOYSSON-BARDIES, B. \& VIHMAN, M.M. (1991). Adaptation to language:Evidence from babbling and first words in four languages. Language, 67 (2), 297-319

DAVIS,B. \& MACNEILAGE, P. (1995). The Articulatory Basis of Babbling. Journal of Speech and Hearing Research, 38: 1199-1211.

DAVIS, B., MACNEILAGE, P., GILDERSLEEVE-NEWMANN C. \& TEIXEIRA, E.R. (1999). Crosslanguage studies of consonant-vowel co-occurrence constraints in infants and adults: Ambient language effects in first words. In: Resumos do Twentieth Annual Child Phonology Conference. University of Wales, Bangor, País de Gales, 18.

FENSON, L.; DALE, P.; REZNICK, D.; BATES, E.; HARTUNG, J. PETHICK, S. \& REILLEY, J. (1992). MacArthur Communicative Development Inventory. San Diego, Cal.: Singular Publishing Group. 
GILDERSLEEVE-NEUMANN, C. (2001). Production vs. Ambient language influences on speech development in Quichua, Tese de Doutorado em preparação University of Texas, Austin.

MACNEILAGE, P.F. \& DAVIS, B.L. (1996). From Babbling to First Words: Phonetic Patterns. First ESCA Tutorial and Research Workshop on Speech Producion Modeling. Austrans, France.

MACNEILAGE, P.F. \& DAVIS, B.L. (2000). Origin of the Internal Structure of Word Forms, Science, Vol. 288, 527-531.

MACNEILAGE, P. DAVIS, B., KINNEY, A. \& MATYEAR, C.L. (1999). Origin of Output complexity in infants and in language. Psychological Review.

TEIXEIRA, R.E. (1997). 'The MacArthur Inventory (CDI) Adapted to Brazilian Portuguese: Analysis of the Early Sound Patterns of Children's Targets'- Conferência apresentada ao Speech Production Lab,University of Texas, Austin.

TEIXEIRA, R.E. \& Davis, B. (1999). Phonetic patterns and Ambient Language influences in the speech acquisition of two Brazilian Portuguese Speakers. In: Resumos do VIII International Congress for the Study of Child Language. Universidade do País Basco, San Sebastian, Espanha, 215.

TEIXEIRA, E.R. \& DAVIS, B. (2001). Phonetic patterns and Ambient Language influences in the speech acquisition of two Brazilian Portuguese Speakers. Language and Speech (no prelo). 